dysmorphic features, skeletal anomalies, goitre or pigmentation. The rest of the examination was unrevealing.

Investigations showed normal CBC, ESR, and liver and renal and thyroid functions. Sweat chloride test, and tissue trans-glutaminase concentrations were normal. His IGF-I level $=70$ (IGF-I SDS $=-1$ ) and his bone age $=3$ years. The peak GH response to clonidine stimulation test $=10 \mathrm{ng} / \mathrm{dl}$. Brain MRI showed normal pituitary gland.

Because of the decelerated growth and marginally low IGF-I a trial of GH therapy was started $(0.035 \mathrm{mg} / \mathrm{kg} /$ day s.c. HS). A rapid catch-up of growth occured during the first 3 years of treatment that was maintained at a slower pace during the following 5 years. Testicular enlargement started at 11 years and at 12 years his HtSDS $=0.8$ and bone age $=12.5$ years. His predicted adult height $=181 \mathrm{~cm}$.

Conclusion Prolonged GH treatment of this boy (normal GH-IGF-I axis) with GH unexpectedly resulted in a HTSDS which surpassed his MPHtSDS by $1.4 \mathrm{SD}$.

\section{PO-0073 WITHDRAWN}

\section{PO-0074 PSEUDO-BARTTER'S SYNDROM AS A FIRST MANIFESTATION OF CYSTIC FIBROSIS IN INFANCY}

S Fustik, T Jakovska, L Spirevska. Center for Cystic Fibrosis, University Children's Hospital, Skopje, Macedonia

10.1136/archdischild-2014-307384.744

Background and aims Metabolic alkalosis in association with low serum electrolyte concentration (hyponatremia, hypochloremia, and hypokalemia) is known complication of cystic fibrosis $(\mathrm{CF})$ in infancy. The condition is a metabolic mimickery of Bartter's syndrome, therefore is sometimes referred to as pseudoBartter's syndrome in CF. The aims of study were to estimate the prevalence of this metabolic disorder as a first manifestation of $\mathrm{CF}$ and the influence of some clinical and genetic factors on its developing.

Methods The records of all 85 newly diagnosed infants with CF in the period from 1998 to 2013 were reviewed. In addition to data of acid-base and electrolyte status at first admission, clinical and CFTR genotype data of patients with pseudo-Bartter's syndrome were analysed.

Results 16 infants had manifestations of hyponatremic/hypochloremic dehydration with metabolic alkalosis, therefore the prevalence of pseudo-Bartter's syndrome among newly diagnosed infants with CF in our region was $18.8 \%$. Mean age of patients was 3.5 (range 1-8) months. Most of them were breastfed. Mean values of blood $\mathrm{pH}$, serum bicarbonate, sodium, chloride and potassium $(\mathrm{mmol} / \mathrm{L})$ were: $7.57 \pm 0.06,44.89 \pm$ $7.23,117.87 \pm 5.38,67.06 \pm 8.48,2.69 \pm 0.47$, respectively. Concerning CFTR genotypes of these patients, a great variability was found: F508del/ F508del (7), F508del/G542X (3), F508del/ $621+\mathrm{G}>\mathrm{T}(1), \mathrm{F} 508 \mathrm{del} / 457 \mathrm{TAT}>\mathrm{G}(1), \mathrm{F} 508 \mathrm{del} / 711+3 \mathrm{~A}>\mathrm{G}$ (1), G126D/V456F(1) and F508del/Unknown (2). Three of them were pancreatic sufficient.

Conclusions The possibility of CF should be seriously considered in any infant with metabolic alkalosis and hypoelectrolytemia, whether or not there are associated pulmonary and/or gastrointestinal symptoms. The initial diagnosis of Bartter's syndrome can be excluded by hypochloruria.

\section{P0-0075 HEAD CIRCUMFERENCE IN THE FIRST YEAR AFTER BIRTH - THE EFFECT OF PRENATAL EXPOSURE TO ORGANOCHLORINES AND PHTHALATES}

${ }^{1} \mathrm{M}$ de Cock, ${ }^{2} \mathrm{MR}$ de Boer, ${ }^{3} \mathrm{M}$ Lamoree, ${ }^{3} \mathrm{~J}$ Legler, ${ }^{1} \mathrm{M}$ van de Bor. ${ }^{1}$ Health and Life Sciences, VU University, Amsterdam, Netherlands; ${ }^{2}$ Health Sciences, VU University, Amsterdam, Netherlands; ${ }^{3}$ Institute for Environmental Studies, VU University, Amsterdam, Netherlands

\subsection{6/archdischild-2014-307384.745}

Background Children with autism may experience increased head growth during the first year of life. Furthermore, early life exposure to endocrine disrupting chemicals (EDCs) may be associated with an increased risk of autism. Exposure to EDCs has been associated with fetal head circumference (HC), however research on long-term effects is scarce.

Objective To assess the association between prenatal exposure to various EDCs and HC in the first year of life.

Methods Cord plasma or breast milk was used to determine exposure to amongst others dichlorodiphenyldichloroethylene (DDE), mono (2-ethyl-5-hydroxyhexyl)phthalate (MEHHP), and mono (2-ethyl-5-carboxypentyl)phthalate (MECPP). Data on HC until 11 months after birth was obtained. Mixed models were composed for each compound. Exposure quartiles, time, and gender were added to the models as fixed effects. Subject was added as a random effect.

Results Boys with high MECPP exposure had a consistently higher HC than lower exposed boys ( $p=0.047)$. This was also observed for girls in Q2 for both MEHHP ( $p=0.018$ ) and DDE $(p<0.001)$ exposure. For MEHHP the difference with the quartile showing the smallest $\mathrm{HC}$ was $2 \mathrm{~cm}$ at 11 months of age, which was not statistically significant (HC Q2: 44.9, 95\% CI 43.2-46.7; HC Q3: 42.9, 95\% CI 42.2-43.5).

Conclusion For MECPP, MEHHP, and DDE exposure over time, a higher $\mathrm{HC}$ was observed in low exposed children in a gender-specific way. Follow-up is warranted to see if associations persist into later childhood and are related to autism spectrum disorders.

\section{PO-0076 PREVALENCE OF OVERWEIGHT AND OBESITY AMONG 5-6-YEAR-OLD CHILDREN IN NORTH PART OF SERBIA}

S Turudic. Department of Pediatrics, Local Health Center "Dr Milorad Mika Pavlovic", Indjija, Serbia

\subsection{6/archdischild-2014-307384.746}

Background and aims Overweight and obesity among children became an important public health concern as their prevalence within the preschool population has been markedly increased in last decades. The aim of the present study was to examine and compare the prevalence of overweight and obesity among 5-6 year old children either sex in year 2003 and year 2013.

Methods Overweight and obesity were measured by body mass index (BMI). BMI 85-95th percentile was considered as overweight and BMI $\geq 95$ th percentile represents obesity. The study was performed in the total population of 859 nonselected 5-6 year old children (423 in 2003 and 436 in 2013) by retrospective analysis of their medical documentations.

Results The overall prevalence of overweight in 2003 was $9.63 \%$, whereas the prevalence of obesity was $9.46 \%$. However, 10 years later, in 2013, the prevalence of overweight was significantly higher $(15.6 \%)$ as well as the prevalence of obesity $(13.53 \%)$. Prevalence of overweight and obesity among 